\title{
ON THE HOMOMORPHISMS OF LOCALLY COMPACT GROUPS ${ }^{1}$
}

\author{
D. H. LEE
}

\begin{abstract}
In this paper, we establish a conjugacy theorem of homomorphisms of a locally compact connected semisimple group into a locally compact group.
\end{abstract}

For locally compact groups $G$ and $H$, let $\operatorname{Hom}(G, H)$ denote the space of all homomorphisms of $G$ into $H$ under the compact open topology. $f_{1}, f_{2} \in \operatorname{Hom}(G, H)$ are said to be conjugate if there exists $h \in H$ such that $f_{2}(x)=h f_{1}(x) h^{-1}$ for all $x \in G$. For papers concerning various types of conjugacy of homomorphisms, we refer to [3], [4], [5], [6], [7], [9], and [10]. In [6] and [7], it is shown that two homomorphisms of compact $G$ are conjugate by an element of the identity component $H_{0}$ of $H$ if and only if they are in the same connected component of the space $\operatorname{Hom}(G, H)$. For noncompact $G$, however, the situation seems less favorable as the character groups of locally compact abelian groups show. Thus it seems natural to ask whether the similar conjugacy theorem holds for groups in which there are no nontrivial connected normal abelian subgroups. The main purpose of this paper is to establish the conjugacy for the above mentioned groups.

In our approach to the problem, we adopt the point of view in the theory of deformation of homomorphisms of Lie groups ([9], [10]). §1 contains basic materials for later use. In $\S 2$, we present the proof of the rigidity theorem of Nijenhuis and Richardson [9, Theorem C], for the sake of completeness. (Their proof does not seem to have appeared.) $\S 3$ is devoted for the proof of the main theorem (Theorem 2 in $\S 3$ ).

1. Basic definitions and conventions. (1.1) Let $G$ be a locally compact group and let $\rho$ be a continuous representation of $G$ in a finite-dimensional real vector space $V$. A continuous map $\varphi: G \rightarrow V$ is called a 1-cocycle of $G$ with values in $V$ (relative to $\rho$ ) if, for $x, y \in G, \varphi(x y)=\varphi(x)+\rho(x)(\varphi(y))$.

Received by the editors January 4, 1971.

AMS (MOS) subject classification. (1969). Primary 2210, 2220, 2250.

Key words and phrases. Conjugate, exponential map, cocycles, coboundaries, rigidity, semisimple, projective limit, centralizer.

${ }^{1}$ This research is supported in part by NSF Grant GP-21180.

c) American Mathematical Society 1973 
The set of all 1-cocycles with values in $V$ forms a vector space, which we denote by $Z^{1}(G, V, \rho)$. A cocycle $\varphi \in Z^{1}(G, V, \rho)$ is called a 1-coboundary, if there exists $v \in V$ such that $\varphi(x)=\rho(x)(v)-v$. The set of all 1-coboundaries forms a subspace $B^{1}(G, V, \rho)$. Let

$$
H^{1}(G, V, \rho)=Z^{\prime}(G, V, \rho) / B^{1}(G, V, \rho),
$$

the 1-cohomology space of $G$ with coefficients in $V$. For the detailed discussion, see [8].

(1.2) Let $G$ and $H$ be locally compact groups. Then the set $\operatorname{Hom}(G, H)$ becomes a topological space under the compact-open topology. To describe neighborhoods of $f_{0} \in \operatorname{Hom}(G, H)$, let $C$ be a compact subset of $G$ and let $U$ be a 1-neighborhood in $H$. Then define

$$
W\left(C, U ; f_{0}\right)=\left\{f \in \operatorname{Hom}(G, H): f(x) f_{0}(x)^{-1} \in U \text { for all } x \in C\right\} .
$$

When $C$ and $U$ run over all compact subsets of $G$ and all 1-neighborhoods in $H$, respectively, the sets $W\left(C, U ; f_{0}\right)$ form a neighborhood basis of $f_{0}$ in $\operatorname{Hom}(G, H)$. If $G$ and $H$ are connected Lie groups, then we may embed $\operatorname{Hom}(G, H)$ into $\operatorname{Hom}(\mathscr{G}, \mathscr{H})$ by $f \rightarrow d f$, where $d f: \mathscr{G} \rightarrow \mathscr{H}$ is the differential of $f$ at $1, \mathscr{G}$ and $\mathscr{H}$ being identified with the tangent linear spaces of $G$ and $H$, respectively, at 1 . For simply connected $G$, this embedding is a homeomorphism.

(1.3) The following notation and convention are standard throughout this paper. For any topological group $H, H_{0}, Z(H)$ and $\operatorname{Aut}(H)$ denote the 1 -component, the center and the automorphism group of $H$, respectively. Also, for $x \in H, I_{x}$ denotes the inner automorphism of $H$ induced by $x$, and, for $X \subset H$, Int $(X)=\left\{I_{x} ; x \in X\right\}$ and $Z_{H}(X)$ is the centralizer of $X$ in $H$. When $H$ is a Lie group, $\operatorname{Ad}_{H}$ denotes the adjoint representation of $H$ in its Lie algebra. A connected Lie group and its Lie algebra are denoted by the same capital italic and capital English script letters, respectively. Thus, for example, if $G$ is a Lie group, then $\mathscr{G}$ denotes the Lie algebra of $G$.

2. On rigidity of homomorphisms of Lie groups. In this section we prove the announced result of [9] on rigidity of homomorphisms of Lie groups and extend it to locally compact groups.

(2.1) Let $G$ be a connected Lie group with $\operatorname{dim} G=n$ and let $\sigma: \widetilde{G} \rightarrow G$ be the universal covering group of $G$. Then, for any Lie group $H$, $\sigma^{*}=\operatorname{Hom}(\sigma, 1): \operatorname{Hom}(G, H) \rightarrow \operatorname{Hom}(\tilde{G}, H)$ is an embedding, and $\tilde{f} \in$ $\operatorname{Hom}(\tilde{G}, H)$ is in the image of $\sigma^{*}$ if and only if $\tilde{f}$ is trivial on $\operatorname{Ker}(\sigma)$. Then $\operatorname{Ker}(\sigma)$ is finitely generated (see, for example, Hochschild, Structure of Lie groups, p. 189, Theorem 1.2). Let $\left\{\tilde{b}_{1}, \cdots, \tilde{b}_{m}\right\}$ be a generating set for $\operatorname{Ker}(\sigma)$. Then $\tilde{f} \in \operatorname{Im}\left(\sigma^{*}\right)$ if and only if $\tilde{f}\left(\tilde{b}_{j}\right)=1,1 \leqq j \leqq m$.

Now we choose a basis $\left\{X_{1}, \cdots, X_{n}\right\}$ for $\mathscr{G}$ and let $\left\{x_{1}, \cdots, x_{n}\right\}$ be a canonical system of coordinates for a fixed exponential map $\exp _{G}: \mathscr{G} \rightarrow \tilde{G}$ 
defined on a 1-neighborhood $V$ of $\tilde{G}$. (See [1, p. 118].) Thus if $a \in V$, then $a=\exp \left(\sum_{k=1}^{n} x_{k}(a) X_{k}\right)$, where $x_{k}(a)$ denotes the $k$ th coordinate. Since $V$ generates $\tilde{G}$, for each $j$, there exists $b_{j, 1}, \cdots, b_{j, m(j)} \in V$ such that

$$
\tilde{b}_{j}=\prod_{p=1}^{m(j)} b_{j, p}=\prod_{p=1}^{m(j)} \exp _{G}\left(\sum_{k=1}^{n} x_{k}\left(b_{j, p}\right) X_{k}\right) .
$$

Now we identify $\operatorname{Hom}(\tilde{G}, H)$ with $\operatorname{Hom}(\mathscr{G}, \mathscr{H})$ under $\stackrel{\theta}{\rightarrow} d f$, where $d f$ denotes the differential of $f$ at $1, \mathscr{G}, \mathscr{H}$ being identified with tangent linear spaces of $G$ and $H$, respectively, at 1 .

Thus we have an embedding $\theta \cdot \sigma^{*}: \operatorname{Hom}(G, H) \rightarrow \operatorname{Hom}(\mathscr{G}, \mathscr{H})$. On the other hand, every homomorphism $\varphi \in \operatorname{Hom}(\mathscr{G}, \mathscr{H})$ is uniquely determined by the $\varphi\left(X_{i}\right)=Y_{i}$ and these $Y_{i}$ can be chosen arbitrarily provided they satisfy

$$
\sum_{k=1}^{n} c_{i j k} Y_{k}-\left[Y_{i}, Y_{j}\right]=0, \quad 1 \leqq i, j \leqq n,
$$

where the $c_{i j k}$ are structural constants of $\mathscr{G}$. Hence if we define $\varepsilon: \operatorname{Hom}(G, H) \rightarrow \mathscr{H}^{n}, \mathscr{H}^{n}$ being the product of $n$ copies of $\mathscr{H}$, by $\varepsilon(f)=$ $\left(d f\left(X_{1}\right), \cdots, d f\left(X_{n}\right)\right)$, then we have, using $f \cdot \exp _{G}=\exp _{H} \cdot d f$ in (1).

LEMMA 1. $\left(Y_{1}, \cdots, Y_{n}\right) \in \mathscr{H}^{n}$ is in $\operatorname{Im}(\varepsilon)$ if and only if the following hold

$$
\begin{aligned}
\prod_{p=1}^{n(j)} \exp _{H}\left(\sum_{k=1}^{n} x_{k}\left(b_{j, p}\right) Y_{k}\right)=0, & 1 \leqq j \leqq m, \\
\sum_{j}^{n} c_{i j k} Y_{k}-\left[Y_{i}, Y_{j}\right]=0, & 1 \leqq i, j \leqq n .
\end{aligned}
$$

Define $\Phi_{j}\left(Y_{1}, \cdots, Y_{n}\right)$ and $\Psi_{i, j}^{\circ}\left(Y_{1}, \cdots, Y_{n}\right)$ to be the left-hand side of (i) and (ii), respectively. Then $\Phi_{j}, 1 \leqq j \leqq m ; \Psi_{i, j}, 1 \leqq i, j \leqq n$, are all $C^{\infty}$-maps of $\mathscr{H}^{n}$ into $\mathscr{H}$.

(2.2) Let $\rho: G \rightarrow \operatorname{Aut}(V)$ be a finite-dimensional real representation. Since $\left\{X_{1}, \cdots, X_{n}\right\}$ is a basis of $\mathscr{G}$, we have an embedding $\varepsilon^{\prime}: Z^{1}(G, V, \rho) \rightarrow$ $V^{n}$ defined by $\varepsilon^{\prime}(f)=\left(d f\left(X_{1}\right), \cdots, d f\left(X_{n}\right)\right)$. In the sequel we give a convenient description of $\operatorname{Im}\left(\varepsilon^{\prime}\right)$.

For this purpose, we take the semidirect product $V \times{ }_{\rho} G$ of the vector group $V$ by $G$ relative to $\rho$. Then for $f \in Z^{1}(G, V, \rho), f^{\prime}: G \rightarrow V \times{ }_{\rho} G$ defined by $f^{\prime}(g)=(f(g), g), g \in G$, is a homomorphism. Hence we can apply Lemma 1 to $f^{\prime}$ to get

Lemma 2. $\left(v_{1}, \cdots, v_{n}\right) \in V$ is in $\operatorname{Im}\left(\varepsilon^{\prime}\right)$ if and only if

$$
\sum_{p=1}^{m(j)} \sum_{k=1}^{n} \delta_{j, p, k}=0, \quad 1 \leqq j \leqq l,
$$


where $\delta_{j, p, k}=x_{k}\left(b_{j, p}\right) \rho\left(b_{j, 1} \cdots b_{j, p-1}\right) v_{k}(1 \leqq k \leqq n, 1 \leqq p \leqq m(j), 1 \leqq j \leqq m)$

(ii) $\left(\sum_{k}^{n} c_{i j k} X_{k}\right)-d \rho\left(X_{j}\right)\left(v_{i}\right)+d \rho\left(X_{i}\right)\left(v_{j}\right)=0 \quad(1 \leqq i, j \leqq n)$,

where $d \rho: \mathscr{G} \rightarrow \operatorname{End}(V)$ is the differential of $\rho$.

(2.3) With this preparation, we can prove:

TheOREM (SEE [9]). Let $G$ be a connected Lie group and let $f_{0} \in$ $\operatorname{Hom}(G, H), H$ any Lie group. If $H^{1}\left(G, \mathscr{H}, \operatorname{Ad} \circ f_{0}\right)=0$, then $\operatorname{Int}\left(H_{0}\right) \circ f_{0}$ is a neighborhood of $f_{0}$.

Proof. We first identify $\operatorname{Hom}(G, H)$ with a closed subset of $\mathscr{H}^{n}$ under $\varepsilon(2.1)$.

Then we can identify the tangent linear space of $\mathscr{H}^{n}$ at $f_{0}$ with that of $\mathscr{H}^{n}$ at $(0, \cdots, 0)$ by the right translation. On the other hand, we identify the tangent linear space of $\mathscr{H}^{n}$ at $(0, \cdots, 0)$ with $\mathscr{H}^{n}$.

Now we define $\chi: H \rightarrow \mathscr{H}^{n}$ by $\chi(h)=I_{h} \circ f_{0}, h \in H$. Then it is easy to see, using Lemma 2 in (2.2), that

$$
\begin{aligned}
\operatorname{Im}(d \chi) & =\varepsilon^{\prime}\left(B^{1}\left(G, \mathscr{H}, \operatorname{Ad} \circ f_{0}\right)\right), \\
\left(\bigcap_{j}^{m} \operatorname{Ker}\left(d \Phi_{j}\right)\right) \cap\left(\bigcap_{i, j}^{n} \operatorname{Ker}\left(d \Psi_{i, j}\right)\right) & =\varepsilon^{\prime}\left(Z^{1}\left(G, \mathscr{H}, \operatorname{Ad} \circ f_{0}\right)\right)
\end{aligned}
$$

where $d \Phi_{j}, d \Psi_{i, j}$ denote the differentials of $\Phi_{j}, \Psi_{i, j}$, respectively at $f_{0}$ and $d \chi$ the differential of $\chi$ at 1 .

We thus apply Lemma 1 of Weil [10] to the space $H$ and $\mathscr{H}^{n}$ and the maps $\chi$ and $\left\{\Phi_{j}, \Psi_{i, j}\right\}$ to find an open neighborhood $U$ of 1 in $H$ such that $\chi(U)$ is open. But $f_{0} \in \chi(U) \subset \operatorname{Int}(H) \cdot f_{0}$, which proves Theorem 1 .

We generalize Theorem 1 to locally compact groups as follows:

THEOREM 1'. If $G$ is a connected locally compact group, then under the same hypothesis of Theorem 1 the conclusion of Theorem 1 holds.

Proof. Since $H$ is a Lie group, there exists an open 1-neighborhood $V$ which contains no nontrivial subgroup of $H$. By the hypothesis, the 1neighborhood $f_{0}^{-1}(V)$ contains a compact normal subgroup $K$ such that $G / K$ is a Lie group. Then for $\varphi \in Z^{1}\left(G, \mathscr{H}, \operatorname{Ad} \circ f_{0}\right), \varphi(K)=0$ and thus if we define $\varphi^{*}: G / K \rightarrow \mathscr{H}$, by $\varphi^{*}(x K)=\varphi(x), x \in G$, then

$$
\varphi^{*} \in Z^{1}\left(G / K, \mathscr{H}, \text { Ad } \circ f_{0}^{*}\right),
$$

where $f_{0}^{*} \in \operatorname{Hom}(G / K, H)$ is induced by $f_{0}$.

The map $\varphi \rightarrow \varphi^{*}$ induces an isomorphism

$$
H^{1}\left(G, \mathscr{H}, \operatorname{Ad} \circ f_{0}\right) \cong H^{1}\left(G / K, \mathscr{H}, \text { Ad } \circ f_{0}^{*}\right) .
$$


Thus under our hypothesis $H^{1}\left(G \mid K, \mathscr{H}, \operatorname{Ad} \circ f_{0}^{*}\right)=0$, and by Theorem 1 , there exists an open neighborhood $U^{*}$ of $f_{0}^{*}$ such that $U^{*} \subseteq \operatorname{Int}\left(H_{0}\right) \cdot f_{0}^{*}$.

We now consider the neighborhood $W\left(K, V ; f_{0}\right)$. As $f_{0}(K)=1$, it follows that $f(K)=1$ for all $f \in W\left(K, V ; f_{0}\right)$. Hence, every $f \in W\left(K, V ; f_{0}\right)$ induces $f^{*} \in \operatorname{Hom}(G / K, H)$, and $f \rightarrow f^{*}$ is continuous. Put

$$
U=\left\{f \in W\left(K, V ; f_{0}\right) \mid f^{*} \in U^{*}\right\} .
$$

Then $U$ is an open neighborhood of $f_{0}$ and is contained in $\operatorname{Int}\left(H_{0}\right) \cdot f_{0}$, proving that $\operatorname{Int}\left(H_{0}\right) \cdot f_{0}$ is a neighborhood $f_{0}$.

COROLLARY. Let $G$ be a connected locally compact group and assume that $H^{1}(G, V, \rho)=0$ for all finite-dimensional representations $\rho: G \rightarrow$ $\operatorname{Aut}(V)$. Then, for any Lie group $H$, the connected components in $\operatorname{Hom}(G, H)$ are open and are of the form $\operatorname{Int}\left(H_{0}\right) \cdot f, f \in \operatorname{Hom}(G, H)$.

Proof. Let $\mathscr{C}$ be the connected component of $f_{0}$. Then, for $f \in \mathscr{C}$, $\operatorname{Int}\left(H_{0}\right) \cdot f$ is a connected neighborhood of $f$. Hence $\operatorname{Int}\left(H_{0}\right) \cdot f \subseteq \mathscr{C}$ and $\left\{\operatorname{Int}\left(H_{0}\right) \cdot f \mid f \in \mathscr{C}\right\}$ is a cover of $\mathscr{C}$, from which it follows that $\mathscr{C}$ is open in $\operatorname{Hom}(G, H)$. To prove the second assertion, for each $f \in \mathscr{C}$, there exist $f_{n}$, $n=1,2, \cdots, n$, in $\mathscr{C}$ such that and

$$
\operatorname{Int}\left(H_{0}\right) \cdot f_{i} \cap \operatorname{Int}\left(H_{0}\right) \cdot f_{i+1} \neq \varnothing, \quad i=0,1, \cdots, n-1,
$$

$$
\operatorname{Int}\left(H_{0}\right) \cdot f_{n} \ni f \text {. }
$$

Now the assertion is clear.

3. Proof of the main theorem. In this section we establish the conjugacy theorem (Theorem 2) for semisimple groups.

(3.1) Definition. A locally compact group $G$ is called semisimple, if its radical (that is, the maximal connected solvable normal subgroup, see [5]) of $G$ is trivial.

It is clear from the definition that the center of a semisimple group is totally disconnected and that every closed normal subgroup of a semisimple group is again semisimple.

LEMMA A. Let $G$ be a locally compact semisimple group and let $D$ be a closed totally discrete normal subgroup of $G$. Then $G / D$ is semisimple.

Proof. Let $R^{\prime}$ be the radical of $G / D$ and let $R$ be the inverse image of $R^{\prime}$ under $G \rightarrow G / D$. Then, since $D$ is central in $G, R$ is a closed solvable normal subgroup of $G$. Hence $R_{0}$ is contained in the radical of $G$ which is trivial. Thus $R$ is totally disconnected. Hence $R^{\prime}$ is trivial, proving that $G / D$ is semisimple. 
LEMMA B. A locally compact connected semisimple group is a projective limit of semisimple Lie groups.

Proof. By the structure of a connected locally compact group, it suffices to prove that if $K$ is a compact normal subgroup of $G$, then $G / K$ is semisimple. By a theorem of Iwasawa [5, Theorem 2, p. 515], $G=K \cdot Z_{G}(K)$, which implies that $G / K \cong Z_{G}(K) / Z(K) . Z_{G}(K)$ is semisimple as a closed normal subgroup of $G$. Also $Z(K)$ is totally disconnected. Hence $Z_{G}(K) / Z(K)$ is semisimple by Lemma $\mathrm{A}$; hence $G / K$ is semisimple.

(3.2) Lemma. Let $G$ be a locally compact connected semisimple group. Then the commutator subgroup $G^{\prime}$ of $G$ is dense in $G$.

Proof. Let $\left\{K_{\lambda}\right\}_{\lambda \in \Lambda}$ be a family of compact normal subgroups of $G$ such that $G / K_{\lambda}$ are all Lie groups, and that $G=\operatorname{proj} \lim G / K_{\lambda}$ (by Lemma B of (3.1)). Since the assertion is true for connected Lie groups, we have $G=\bar{G}^{\prime} \cdot K_{\dot{\lambda}}, \lambda \in \Lambda$, where $\bar{G}^{\prime}$ is the closure of $G^{\prime}$. Hence for any $g \in G$, there exists $g_{\lambda} \in \bar{G}^{\prime}$ such that $g=g_{\lambda} k_{\lambda}$, for some $k_{\lambda} \in K_{\lambda}$. Let $\left\{k_{\lambda_{i}}\right\}_{i}$ be a converging subnet of the net $\left\{k_{\lambda}\right\}_{\lambda \in \Lambda}$. Then $\lim _{i} g_{\lambda_{i}}$ exists and is equal to $g$, proving that $g \in \bar{G}^{\prime}$. Hence $G=\bar{G}^{\prime}$.

(3.3) LEMMA. Let $K$ be a compact normal subgroup of a connected semisimple locally compact group $G$ and let $\pi: G \rightarrow G / K$ be the natural map. Then, for any connected subset $X$ of $G$ containing $1, \pi: Z_{G}(X) \rightarrow Z_{G / K}(\pi(X))$ is onto.

Proof. We can write $G=K \cdot Z_{G}(K)$. (See [5].) Then for $z^{*} \in$ $Z_{G / K}(\pi(X))$, we choose $z \in Z_{G}(K)$ so that $\pi(z)=z^{*}$. Hence, for each $x \in X$,

$$
[z, x]=z x z^{-1} x^{-1} \in K \cap Z_{G}(K)=Z(K) .
$$

But $x \rightarrow[z, x]$ is a continuous map from the connected space $X$ into the totally disconnected $Z(K)$. Hence this map is constant and $1 \in X$ implies that $[z, x]=1$, proving that $z \in Z_{G}(X)$.

(3.4) LEMMA. Let $G$ be a locally compact connected semisimple group. Then $H^{1}(G, V, \rho)=0$ for any finite-dimensional representation $\rho: G \rightarrow \operatorname{Aut}(V)$.

Proof. Assume that $G$ is a Lie group and let $f \in Z^{1}(G, V, \rho)$. Then $f^{\prime}: G \rightarrow V \times{ }_{\rho} G$, the semidirect product of $V$ by $G$ relative to $\rho$, defined by $f^{\prime}(g)=(f(g), g), g \in G$, is a homomorphism. Thus $f^{\prime}(G)$ is a semisimple subgroup of $V \times{ }_{\rho} G$. Since any two maximal semisimple subgroups of a Lie group are conjugate by an element from its radical, we find $v \in V$ 
such that $(v, 1) f^{\prime}(G)(v, 1)^{-1} \subseteq\{0\} \times G$. Hence, for $g \in G, v+f(g)=\rho(g)(v)$, which implies that $f \in B^{1}(G, V, \rho)$, proving the assertion for Lie groups.

Now let $G$ be locally compact and let $U$ be a 1-neighborhood of $\operatorname{Aut}(V)$ which contains no nontrivial subgroup and we choose a compact normal subgroup $K$ in $\rho^{-1}(U)$ such that $G / K$ is a Lie group. Then for every $f \in Z^{1}(G, V, \rho)$, the restriction $f \mid K \in \operatorname{Hom}(K, V)$. Since $K$ is also semisimple, $f \mid K=0$ by (3.2). Thus $f$ induces $f^{*} \in Z^{1}\left(G / K, V, \rho^{*}\right)$, where $\rho^{*}: G / K \rightarrow \operatorname{Aut}(V)$ is induced by $\rho$, and $f \rightarrow f^{*}$ induces an isomorphism $H^{1}(G, V, \rho) \cong H^{1}\left(G / K, V, \rho^{*}\right)$. Since $G / K$ is a Lie group, $H^{1}\left(G / K, V, \rho^{*}\right)=$ 0 , which proves that $H^{1}(G, V, \rho)=0$.

(3.5) Now we are ready to prove the following main theorem of this section.

THEOREM 2. Let $G$ be a locally compact connected semisimple group and $H$ any locally compact group. Then the connected components in $\operatorname{Hom}(G, H)$ are exactly of the form $\operatorname{Int}\left(H_{0}\right) \cdot f, f \in \operatorname{Hom}(G, H)$.

Proof. Since $G$ is connected, it is easy to see that $\operatorname{Hom}(G, H) \cong$ $\operatorname{Hom}\left(G, H_{0}\right)$. Hence we may assume that $H$ is connected. Let $\mathscr{C}$ be any connected component in $\operatorname{Hom}(G, H)$ and fix $f_{0} \in \mathscr{C}$.

Let $R$ denote the radical of $H$ with the descending sequence of the derived groups: $R=R^{(0)} \supset R^{(1)} \supset \cdots \supset R^{(n)} \supset R^{(n+1)}=\{1\}$.

Our proof is based on the induction on $n$, the length of solvability of $R$.

(A) The assertion holds for $n=0$. Note that $H$ in this case is semisimple.

Fix a compact normal subgroup $K$ of $H$ such that $H / K$ is a Lie group, and let $\left\{K_{\lambda}\right\}_{\lambda \in \Lambda}$ be a family of compact normal subgroups with each $K_{\lambda} \subset K$ such that $H / K_{\lambda}$ is a Lie group and that $H$ is a projective limit of $H / K_{\lambda}$.

Thus by (3.4) and the corollary in (2.3), the assertion in the stated theorem holds for $\operatorname{Hom}\left(G, H / K_{\lambda}\right), \lambda \in \Lambda$.

The maps $\operatorname{Hom}(G, H) \rightarrow \operatorname{Hom}\left(G, H / K_{\lambda}\right)$, induced by $H \rightarrow H / K_{\lambda}$, are all continuous. Hence if $f \in \mathscr{C}$, then there exists $h_{\lambda} \in H$ such that, for $x \in G$,

$$
h_{\lambda} f(x) h_{\lambda}^{-1}=f_{0}(x) \bmod K_{\lambda} .
$$

Similarly we find $h_{0} \in H$ such that, for $x \in G, h_{0} f(x) h_{0}^{-1}=f_{0}(x) \bmod K$. Since $K_{\lambda} \subset K, h_{0} f(x) h_{0}^{-1}=h_{\lambda} f(x) h_{\lambda}^{-1} \bmod K$, which implies that $\pi\left(h_{\lambda}^{-1} \cdot h_{0}\right)$ is in the centralizer of $\pi(f(G))$ in $G / K$, where $\pi: G \rightarrow G / K$ is the natural map.

By (3.4), there exists $a \in H$ such that $a$ commutes with every element of $f(G)$ and that $\pi(a)=\pi\left(h_{\lambda}^{-1} h_{0}\right), \lambda \in \Lambda$.

Thus $h_{\lambda} a \in h_{0} K, \lambda \in \Lambda$, and we may hence assume that the $h_{\lambda}$ are all in $h_{0} K$ (by replacing $h_{\lambda}$ by $h_{\lambda} a$ if necessary). Since $h_{0} K$ is compact, the net 
$\left\{h_{\dot{\lambda}}\right\}_{\lambda \in \Lambda}$ has a converging subnet $\left\{h_{\dot{\lambda}_{i}}\right\}_{i}$ with $\lim _{i} h_{\lambda_{i}}=h$. Then we have $I_{h} \circ f(x)=f_{0}(x), x \in G$, which proves (A).

(B) Assume now that the theorem holds for $H$ with the length of solvability of its radical less than $n$, and consider the sequence in the beginning of our proof.

Applying the induction hypothesis to the group $H / R^{(n)}$, we find $h \in H$ such that, for $x \in G, h f(x) h^{-1}=f_{0}(x) \bmod R^{(n)}$. Let $\alpha(x)=h f(x) h^{-1} f_{0}(x)^{-1}$, $x \in G$. Then $\alpha$ is clearly a continuous map of $G$ into $R^{(n)}$. Let $K$ be the maximal compact subgroup of the abelian group $R^{(n)}$. Then $K$, being a characteristic subgroup of $R^{(n)}$, is normal (hence central) in $H$ and $R^{(n)} / K$ is a vector group. Let $\rho(x), x \in G$, be the automorphism of $R^{(n)}$, defined by $y \rightarrow f_{0}(x) y f_{0}(x)^{-1}, y \in R^{(n)}$. Then $\rho$ leaves $K$ pointwise fixed, hence $\rho$ induces a representation $\rho^{*}$ of $G$ in the vector space $R^{(n)} / K$.

Now it is a trivial matter to verify that the composite map $\alpha^{*}: G \stackrel{\alpha}{\rightarrow}$ $R^{(n)} \stackrel{\pi}{\rightarrow} R^{(n)} / K$ is in $Z^{1}\left(G, R^{(n)} / K, \rho^{*}\right)$. But $H^{1}\left(G, R^{(n)} / K, \rho^{*}\right)=0$ by (3.4). Hence there exists $a \in R^{(n)}$ such that

$$
\alpha^{*}(x)=\pi(a)^{-1} \rho^{*}(x)(\pi(a)), \quad x \in G .
$$

From the definition of $\alpha^{*}(x)$, it follows that, for $x \in G, h f(x) h^{-1}=$ $a^{-1} f_{0}(x) a \bmod K$, and thus we have $\beta(x)=a h f(x)(a h)^{-1} f_{0}(x)^{-1} \in K$. An easy calculation using the centrality of $K$ in $H$ shows that $\beta: G \rightarrow K$ is a continuous homomorphism. As $K$ is abelian, it follows that the first derived group $G^{\prime} \subset \operatorname{Ker} \beta$, Since $G^{\prime}$ is dense in $G$ by (3.2), we have that $\beta(G)=1$, which means that $a h f(x)(a h)^{-1}=f_{0}(x)$ for all $x \in G$, proving that $f$ is conjugate to $f_{0}$.

REMARK. Theorem 2 is a generalization of Theorem (4.3) in [3, p. 338].

\section{BIBLIOGRAPHY}

1. C. Chevalley, Theory of Lie groups, Princeton Univ. Press, Princeton, N.J., 1946. MR 7, 412 .

2. M. Goto, Note on a topology of a dual space, Proc. Amer. Math. Soc. 12 (1961), 41-46. MR 23 \#A3195.

3. S. Grosser, O. Loos and M. Moskowitz, Über Automorphismengruppen lokalkompakter Gruppen und Derivationen von Lie-Gruppen, Math. Z. 114 (1970), 321-339. MR 41 \#8575.

4. K. Hofmann and P. Mostert, Die topologische Struktur des Raumes der Epimorphismen kompakter Gruppen, Arch. Math. 16 (1965), 191-196. MR 31 \#5920.

5. K. Iwasawa, On some types of topological groups, Ann. of Math. (2) 50 (1949), 507-558. MR 10, 679.

6. D. H. Lee and T. S. Wu, On conjugacy of homomorphisms of topological groups, Illinois J. Math. 13 (1969), 694-699. MR 40 \#1525.

7. - On conjugacy of homomorphisms of topological groups. II, Illinois J. Math. 14 (1970), 409-413. MR 42 \#1939. 
8. G. D. Mostow, Cohomology of topological groups and solvmanifolds, Ann. of Math. (2) 73 (1961), 20-48. MR 23 \#A2484.

9. A. Nijenhuis and R. W. Richardson, Jr., Deformations of homomorphisms of Lie groups and Lie algebras, Bull. Amer. Math. Soc. 73 (1967), 175-179. MR 34 \#4414.

10. A. Weil, Remarks on the cohomology of groups, Ann. of Math. (2) 80 (1964), 149-157. MR 30 \#199.

Department of Mathematics, Case Western Reserve University, Cleveland, ОНІо 44106 